\title{
Application of CT Image Technology Based on Nearest Neighbor Propagation Clustering Segmentation Algorithm in Lung Cancer Radiotherapy
}

\author{
Weixiang Chen $\mathbb{D}^{1},{ }^{1}$ Jianfu Zhao $\mathbb{D}^{1},{ }^{1}$ Zhenhui Dai $\mathbb{D}^{2},{ }^{2}$ Mingyue Lv $\mathbb{D}^{1},{ }^{1}$ Zhenhua Yang $\mathbb{D}^{1},{ }^{1}$ \\ and Yuqin Zhang ${ }^{1}{ }^{1}$ \\ ${ }^{1}$ Department of Oncology, The First Affiliated Hospital of Jinan University, Guangzhou 510630, China \\ ${ }^{2}$ Department of Radiotherapy, The Second Affiliated Hospital of Guangzhou University of Chinese Medicine, \\ Guangzhou 510120, China
}

Correspondence should be addressed to Yuqin Zhang; 130101140227@stu.nepu.edu.cn

Received 14 May 2021; Accepted 5 October 2021; Published 3 December 2021

Academic Editor: Gustavo Ramirez

Copyright (c) 2021 Weixiang Chen et al. This is an open access article distributed under the Creative Commons Attribution License, which permits unrestricted use, distribution, and reproduction in any medium, provided the original work is properly cited.

\begin{abstract}
Objective. This paper uses the nearest neighbor propagation clustering segmentation algorithm to explore the impact of PET/CT image segmentation technology on lung cancer radiotherapy planning. Methods. In this paper, PET/CT scan was performed on 12 patients with nonmetastatic lung cancer. The self-written automatic segmentation program based on PCNN model is used to segment the PET target area, and then the tumor target area is manually sketched based on CT images and PET/CT images, and the intensity-modulated radiotherapy plan is formulated with the same parameters. Target volume and dose distribution were analyzed. Results. There was no statistical difference between the PET automatic segmentation target area and the PET manual contouring target area $(P<0.05)$; the segmentation method was accurate and reliable; the difference between the CT manual contouring target area was statistically significant $(P 0.05)$. Conclusion. Based on the nearest neighbor propagation clustering segmentation algorithm, PET/CT image segmentation technology improves the accuracy of tumor target area delineation. The radiotherapy plan based on the segmentation target area can reduce the normal tissue exposure range and reduce the incidence of complications.
\end{abstract}

\section{Introduction}

The rapid development of precision radiotherapy technology, three-dimensional conformal radiation therapy (3DCRT), intensity-modulated radiation therapy (IMRT), and other technologies plays a very important role. Target area delineation is the basis of precise radiotherapy. For a long time, radiotherapy mainly used CT to delineate the tumor target area. When lung cancer is accompanied by atelectasis and obstructive pneumonia, CT is difficult to distinguish the exact boundary of the tumor.

PET/CT functional imaging plays an important role in radiotherapy. The PET/CT metabolic target area can guide the outline of the traditional CT target area and accurately define the tumor target area; formulating a radiotherapy plan based on the PET/CT target area can prevent tumor recurrence in the field and reduce normal tissue damage. $\mathrm{PET} / \mathrm{CT}$ can distinguish tumor tissues from surrounding normal lung tissues well and is suitable for lung cancer target area delineation. However, manual segmentation of PET/CT target area is time-consuming and inaccurate, and there is an urgent need for automatic segmentation method of PET/CT image target area in clinic. Pulse-coupled neural network (PCNN) can produce a recognition effect similar to human vision and has a good segmentation effect on low-resolution images. Because PET can provide biological information of lesions, the use of PET images to guide the formulation or revision of radiotherapy plans for non-small-cell lung cancer 
(NSCLC) has become a research hotspot. The accuracy of the fusion of PET images and CT images limits its advantages to a certain extent. The appearance of the same-machine PETCT greatly reduces the mechanical errors of image fusion and the impact of patient position changes. The fusion accuracy of PET-CT may be worse in the lungs than other parts of the body, which may affect the precise positioning of the lesion and the outline of the precise radiotherapy target area. In this paper, an image segmentation technique based on PCNN model is used to segment PET/CT target area, and CT target area is compared to discuss its effect on radiotherapy plan design and normal tissue dose.

\section{Information and Methods}

2.1. Patient Information. We screened 12 patients with lung cancer in our hospital from May 2018 to December 2020, including 7 males and 5 females, aged 52-70 years, with a median age of 61 years. All patients were diagnosed by pathology. The pathological types were 4 cases of squamous cell carcinoma, 7 cases of adenocarcinoma, and 1 case of small cell carcinoma, all without metastasis.

2.2. Image Acquisition. The patient was fasting for more than 6 hours, and the routine measurement of blood glucose concentration was within the normal range. Intravenous injection of $370 \sim 555 \mathrm{MBq} 18 \mathrm{~F}$-deoxyglucose (18F-FDG), resting for 60 minutes in calm, using PET/CT equipment in the same position under calm breathing, full-body CT and PET scans were performed from the top of the head to the sole of the foot. The PET image and the CT image are fused together, and the image data is transmitted to the radiotherapy planning system.

For the imaging, a discovery LS PET-CT instrument was used for regular quality control measurement to ensure that the mechanical fusion accuracy of PET and CT images in each radial direction was within $2.0 \mathrm{~mm}$, and the actual measurement accuracy was within $0.8 \mathrm{~mm}$. The patient was fasted for more than $6 \mathrm{~h}$, intravenously injected FDG5.55 $7.40 \mathrm{MBq} / \mathrm{kg}$, and examined after 60 minutes of rest. During the examination, the patient was immobile and took a calm breathing state. CT scan parameters were $140 \mathrm{kV}, 80 \mathrm{~mA}$, each ring rotation time $0.8 \mathrm{~s}$, and pitch 0.75 . From the top of the head to the pelvic floor, the scanning time was about $47 \mathrm{~s}$, and the reconstructed CT image was $4.25 \mathrm{~mm}$. Immediately after the CT scan, the PET emission scan is performed. Scanning conditions were $144.5 \mathrm{~mm}$ and $4 \mathrm{~min}$ per bed. A total of 5-7 beds were collected from the top of the head to the bottom of the pelvis. There was a layer of overlap between adjacent beds to avoid artifacts. PET images are corrected for attenuation using CT images, and image reconstruction is performed using the ordered subset maximum expected value method (OSEM). All images were analyzed retrospectively by two observers. Two methods are used for the analysis of lesion fusion accuracy: volume method: PET images and CT images are transmitted to the Advantage Work station, and Fusion 1.0.43 software is used to fuse PET and CT images, respectively, and PET cross- sectional images (display conditions: the maximum count value at the lesion is set to $100 \%$, and the lower limit is deducted by $45 \%$ ) and the CT lung window cross-sectional image is delineated layer by layer of interest (VOI) [1]. Volume (VPET) and CT three-dimensional volume (VCT): then outline the total outline of the PET image and CT image of the lesion along the outermost edges of VOIPET and VOICT, named VOIPET + CT, and obtain the total volume of the PET image and CT image of the lesion superimposed together (VPET $+\mathrm{CT})$. The volume of the fusion part of the PET and CT images of the lesion (VPET-CT) can be obtained by the formula VPET-CT $=\mathrm{VPET}+\mathrm{VCT}-\mathrm{VPET}+\mathrm{CT}$ and the percentage of the fusion part volume (VPET-CT) to the total volume (VPET $+\mathrm{CT}) \quad(\mathrm{PPET}-\mathrm{CT}=\mathrm{VPET}-\mathrm{CT} /$ VPET + CT).

\subsection{Method}

2.3.1. Image Segmentation Method. The self-written PCNN segmentation algorithm is used to automatically segment PET/CT images. The target area obtained by segmentation is named GTV-PET auto. The specific steps are as follows: (1) image denoising, (2) setting initial values of PCNN parameters, (3) performing PCNN iteration on the neighborhood of each neuron to obtain segmentation results, (4) determining whether the segmentation results meet the optimal segmentation decision criteria, and (5) taking the segmentation result graph that meets the optimal segmentation decision criterion as the final segmentation result. First calculate the average offset of the current point and move the point to its average offset; then use this as a new starting point to continue moving until it meets certain conditions and ends. The MS algorithm has been successfully applied to the analysis of feature space, and MS has been well applied in image smoothing and image segmentation. The basic principle of the MS algorithm is

$$
M_{h, G}(x) \equiv \frac{\sum_{i=1}^{n} G\left(x_{i}-x / h\right)\left(x_{i}-x\right)}{\sum_{i=1}^{n} G\left(x_{i}-x / h\right)} .
$$

Among them, $G(x)$ is the kernel function, and the Gaussian function is generally used as the kernel function, $x_{i}(i=1,2, \ldots, n)$ is $n$ sample points in $d$ dimensional space $R^{d}, x$ represents the center of the kernel function, and $h$ is the bandwidth. When the MS algorithm performs image smoothing and segmentation, the kernel function bandwidth is an important parameter, which not only determines the number of sampling points participating in the iteration, but also affects the convergence speed and accuracy of the algorithm.

The AP algorithm is clustered according to the similarity between data points, and the similarity matrix is formed by the similarity between $n$ data points. When processing image data, the feature space needs to be defined before calculating the similarity matrix. The features of the image can be selected as the color, texture, statistical characteristics, and shape of the image. In this paper, color information is selected as the main feature. For color images, the color of each point is represented by a three-dimensional vector; then the 
similarity between pixels is defined as the negative Euclidean distance between pixels, that is,

$$
S_{i k}=-\|F(i)-F(k)\|^{2} .
$$

Among them, $F(i), F(k)$ is the color value of point $i$ and point $k$, and the similarity value between points takes negative values. Suppose the image is divided into $m$ regions $R_{i}(i=1,2, \ldots, m)$ after the MS algorithm, and the color vector corresponding to each region is $X_{R_{i}}=\left\{\bar{x}_{1 i}, \bar{x}_{2 i}, \bar{x}_{3 i}\right\}$, where $\bar{x}_{1 i}, \bar{x}_{2 i}, \bar{x}_{3 i}$ is the average of the color components corresponding to all pixels in the itch region. The selection of the time is also important for the ability to accurately merge small regions. In order to obtain good segmentation results, it is necessary to select a color space in which the color difference is related to the Euclidean distance. In this paper, the Luv color model with linear mapping characteristics is used, where $L$ represents brightness, $u$ and $v$, respectively, represent chromaticity coordinates, and the similarity between regions is defined as

$$
S_{i k}=-\left\|X_{R_{i}}-X_{R_{k}}\right\|^{2} .
$$

The AP algorithm introduces a convergent parameter $\lambda$ during information update, called the damping coefficient. The larger the $\lambda$, the faster the convergence speed. At each iteration, the updated results of $r_{i k}$ and $r_{i k}$ are obtained by weighting the updated value in the current iteration process and the result of the previous iteration. Assuming the current iteration number $t$, the weighting formula is

$$
\begin{aligned}
& r_{i k}^{(t)} \longleftarrow(1-\lambda) \times\left(s_{i k}-\max _{k^{\prime} s, t, k^{\prime} \neq k}\left\{a_{i k^{\prime}}^{(t-1)}+s_{i k^{\prime}}\right\}\right)+\lambda \times r_{i k}^{(t-1)}, \\
& a_{i k}^{(t)} \longleftarrow(1-\lambda) \times\left(\min \left\{0, r_{k k}^{(t-1)}+\sum_{t^{\prime} s, t, i^{\prime} \notin\{i, k\}} \max \left\{0, r_{i^{\prime} k}^{(t)}\right\}\right\}\right)+\lambda \times a_{i k}^{(t-1)}, \\
& a_{i k}^{(t)} \longleftarrow(1-\lambda) \times\left(\sum_{t^{\prime} s, t, i^{\prime} \notin\{i, k\}} \max \left\{0, r_{i^{\prime} k}^{(t)}\right\}\right)+\lambda \times a_{i k}^{(t-1)},
\end{aligned}
$$

and among them $\lambda \in(0,1)$.

The MSAP algorithm steps are as follows:

Step 1: use MS algorithm to presegment the input image.

Step 2: calculate the average value of the colors in all areas and use it as the data point input by the AP algorithm.

Step 3: calculate the difference of the color mean of each area to obtain the similarity matrix $\mathrm{S}$.

Step 4: set $p$ and $\lambda$ reasonably $p<0, \lambda \in(0,1)$, and initialize $r_{i k}=0, a_{i k}=0$ to set a maximum number of iterations. Figure 1 shows the flow chart of the algorithm.

In this paper, the value of $p$ is taken as the minimum value or a multiple of the minimum value of the similarity matrix, $p=C \times \min \left(s_{i k}\right)$, where $C$ is a constant and $C>0$. The termination condition of the iterative process is that the number of iterations exceeds the set maximum number of iterations or how many consecutive iterations of the cluster center do not change.

Step 5: according to the similarity matrix and equations (3) to (6), the values of $r_{i k}$ and $a_{i k}$ are calculated and updated iteratively until the iteration termination condition is satisfied, and the final image segmentation result is output.
2.3.2. Outline of Target Area. On the PINNACLEV 9.2 radiotherapy planning system, experienced radiologists delineate the tumor boundary on the cross section layer by layer based on CT images and PET/CT images, and the resulting general tumor target areas are represented as GTVCT (CT manual outline target area) and GTV-PET (PET/CT manual outline target area).

2.3.3. Radiotherapy Plan Design. An experienced physicist uses the PINNACLEV9.2 planning system to develop an IMRT plan based on GTV-CT and GTV-PET auto, respectively. The same parameters are used in the design of the radiotherapy plan. The general tumor target area expands $0.7 \mathrm{~cm}$ outside the GTV boundary to form the clinical target area CTV, and the CTV extension expands $0.6 \mathrm{~cm}$ outside the boundary to form the planned target area PTV. The same isocenter is adopted when making the plan, and all adopt the 5 -field coplanar design. All plans use $6 \mathrm{MV}$ X-ray irradiation of Varian linear accelerator. The prescribed dose is $60 \mathrm{~Gy}$ ( 2 Gy/time, 5 times/week, a total of 30 times). Target dose requirement: $90 \%$ isodose curve surrounds PTV. Dose requirements for organs at risk are as follows: V20 $\leq 30 \%$ in both lungs, Dmax $\leq 40$ Gy in spinal cord, Dmax $\leq 45$ Gy in esophagus, and $\mathrm{D} 1 / 3 \leq 50 \mathrm{~Gy}$ in heart. The dose of the target area and the organs at risk is evaluated by the dose histogram DVH. 


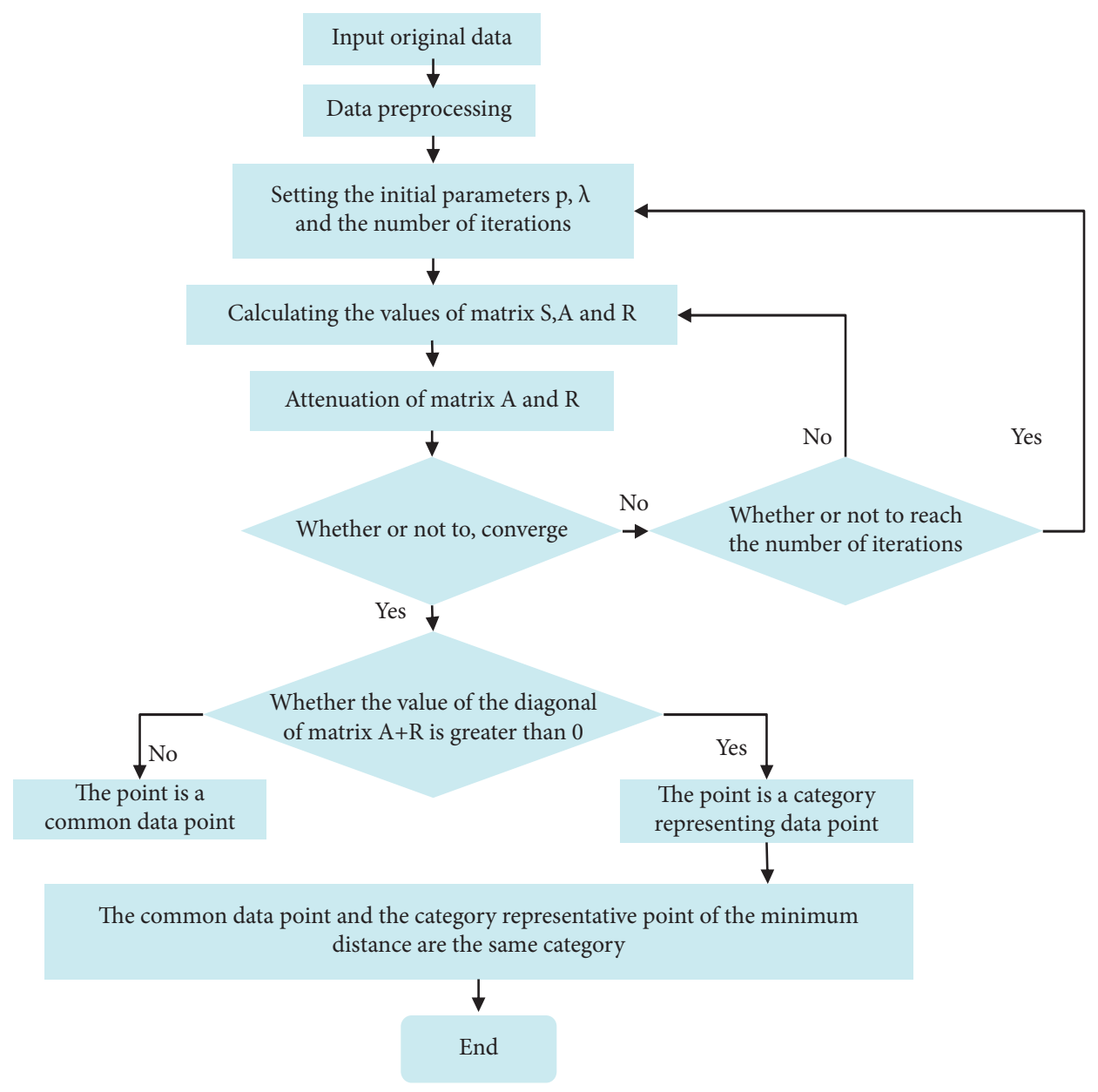

FIGURE 1: MSAP algorithm flowchart.

2.4. Statistical Analysis. SPSS13.0 statistical analysis software was used to perform pairwise $t$-test on the average volume of the GTV target area of three different sketching methods. Paired $t$-test was performed on the dose of radiotherapy plan based on GTV-CT and GTV-PET auto, and $P \leq 0.05$ means there are statistical differences.

\section{Results}

3.1. Comparison of Target Volume. Select one patient's data for explanation. The target areas of GTV-PET auto, GTVPET, and GTV-CT are shown in Figure 2. The red line indicates GTV-PET auto, the yellow line indicates GTVPET, and the green line indicates GTV-CT. It can be seen from the figure that the red and yellow lines almost overlap, and the difference between GTV-PET auto and GTV-PET is small. GTV-PET auto and GTV-PET are both $<\mathrm{GTV}-\mathrm{CT}$.

The software calculates the target volume GTV in three sketching methods, and the average GTV in 12 patients is shown in Table 1 . There was no statistically significant difference between GTV-PET auto and GTV-PET $(P>0.05)$. GTV-PET auto and GTV-PET were both less than GTV-CT, with statistical difference $(P<0.05)$, which further verified the above conclusion.
3.2. Dose Evaluation and Comparison. The dose distribution of the radiotherapy plan based on GTV-CT and GTV-PET auto is shown in Figure 3. It shows the intensity-modulated radiotherapy plan based on the CT target area. Figure 3(b) shows the intensity-modulated radiation therapy plan based on the PET target area. It can be seen that the normal lung tissue intake based on the PET target area is significantly smaller, and the intake of other normal tissues has also decreased.

The normal tissue dose comparison of the two groups of radiotherapy plans is shown in Table 2 . It can be seen from the comparison that when the same dose of $60 \mathrm{~Gy}$ is given to the tumor target area, the PET target area plan is significantly lower than that of the CT target area plan, and the lung tissues V20 and V30 are significantly reduced $(P<0.05)$.

\section{Discussion}

PET/CT, which combines functional image information and anatomical information, is widely used in precise radiotherapy. Compared with the commonly used CT and MR images, PET/CT images are more advantageous in determining tumor range and lymph node metastasis. Studies have shown that PET/CT is used to delineate lung tumor target area, which can reduce GTV when accompanied by 


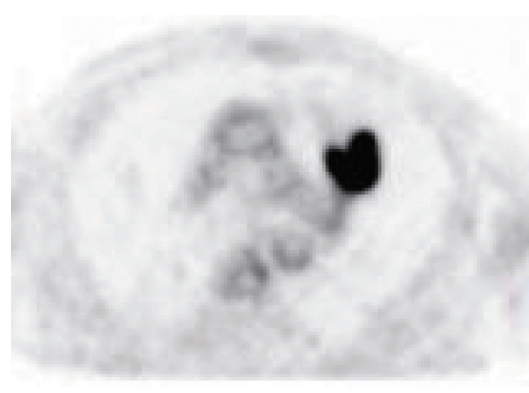

(a)

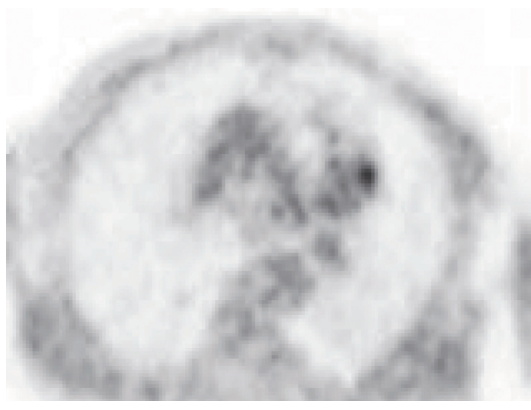

(d)

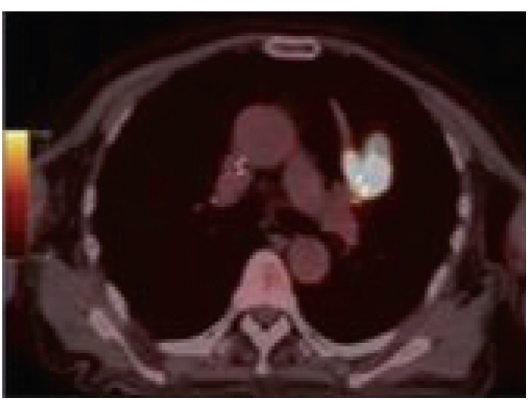

(b)

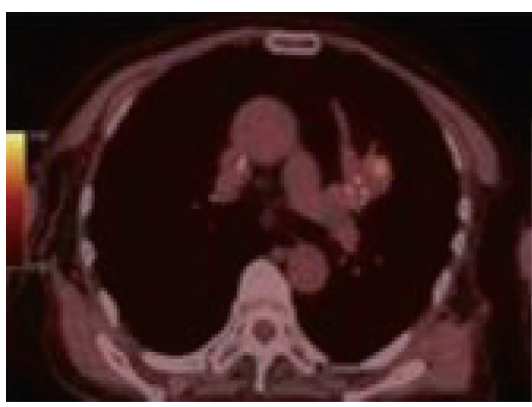

(e)

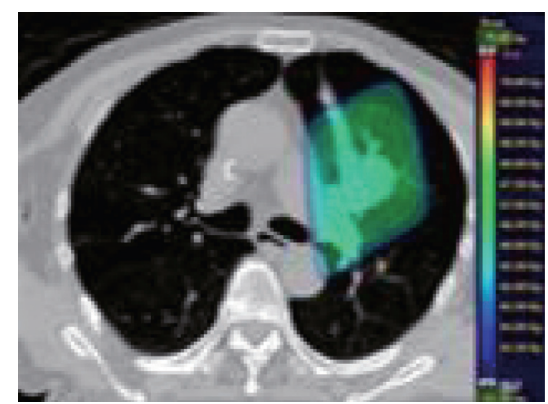

(c)

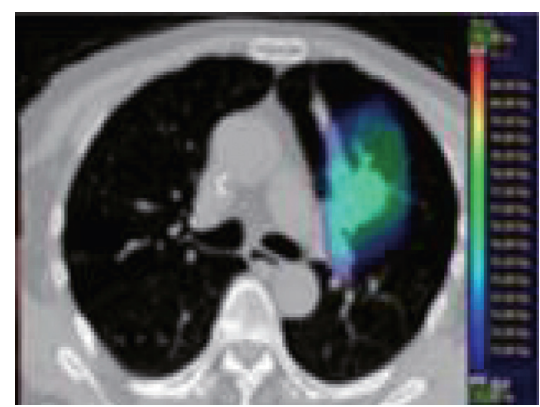

(f)

FIgure 2: The target area outlined in 3 ways. Note. The green line is GTV-CT, the red line is GTV-PET auto, the yellow line is GTV-PET, and the red line and the yellow line almost overlap.

TABLE 1: Comparison of target volume under different sketching methods.

\begin{tabular}{|c|c|c|c|c|}
\hline Sketching method & CT image target volume & PET image target volume & $t$ value & $P$ value \\
\hline Doctor manually sketch & GTV-CT $(161.43 \pm 139.30)$ & GTV-PET $(146.20 \pm 101.32)$ & & \\
\hline $\begin{array}{l}\text { PCNN automatic segmentation } \\
t \text { value } \\
P \text { value }\end{array}$ & 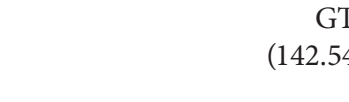 & $\begin{array}{l}\text { auto } \\
\text { 73) } 0.763\end{array}$ & 3.431 & $<0.05$ \\
\hline
\end{tabular}

atelectasis and obstructive pneumonia, thereby better protecting normal lung tissue [2]. By detecting mediastinal lymph node sensitivity higher than CT [3], it can detect metastatic lymph nodes with normal size displayed on CT, making GTV sketch more accurate. The precise radiotherapy plan guided by PET/CT target area is expected to avoid the risk of missed radiation in the target area and can better protect normal tissues, which helps to improve the local control rate of patients and reduce complications.

In recent years, the application of PET and PET-CT in 3DCRT for non-small cell lung cancer has received extensive attention. In particular, the PET-CT all-in-one system has become a new platform for radiotherapy simulation positioning due to its outstanding advantages of the close integration of functional images and anatomical images. Accurate measurement of the fusion accuracy of the lesion is very important to further explore the impact of PET-CT image fusion on the precise radiotherapy target area. The main factors that affect the accuracy of image fusion include differences in image properties; physiological motions of internal organs such as respiratory motion; mechanical errors between PET and CT acquisition systems; movement and differences in patient position during PET and CT acquisition. For the PET-CT all-in-one system, the influence of the latter two factors has been greatly reduced, while the influence of the first two still exists. For PET-CT imaging of lung cancer, breathing mobility is an important influencing factor for image quality and target area delineation. During the PET-CT examination, the PET scan time is relatively long, and the entire chest image is collected for about 4 minutes. The PET image is the superposition of all the information of the lesion during the entire respiratory cycle. Due to the special laws of respiratory movement, in general, the lesion is in the exhaled state longer than the inhaled state, so the PET image may more mainly reflect the information under the exhaled state of the lesion. The CT scan time is very short, and the image of the entire chest is collected for about 8 seconds, which reflects that the information of the respiratory phase at a certain stage of the lesion at that time has a certain relationship with the respiratory frequency and respiratory amplitude of the patient under examination. The randomness of respiratory motion effects may be more pronounced than PET images. The difference in the acquisition phase of the two will cause the PET image and CT image of the lung lesions to not be accurately fused together. When there is a certain fusion error, if the PET-CT fusion image is used to delineate the target area, in order to 
CT

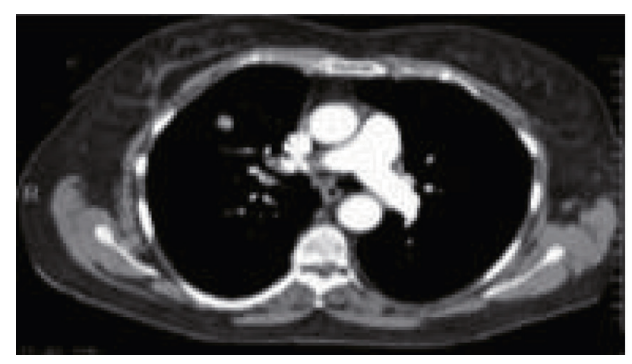

$\mathrm{PET} / \mathrm{CT}$

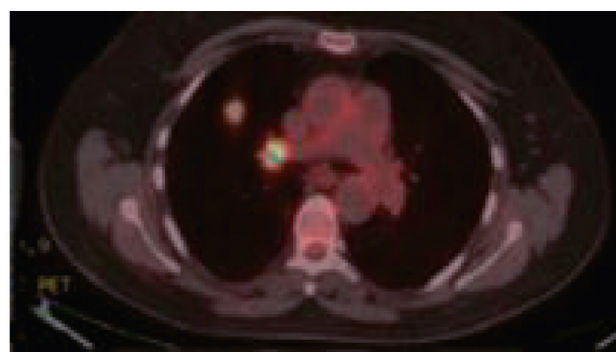

(a)

CT

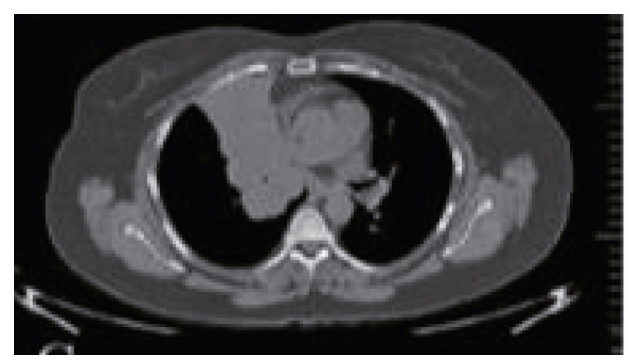

(b)
$\mathrm{PET} / \mathrm{CT}$

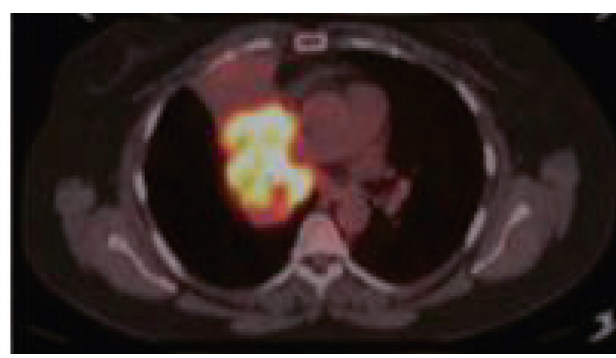

$\mathrm{PET} / \mathrm{CT}$

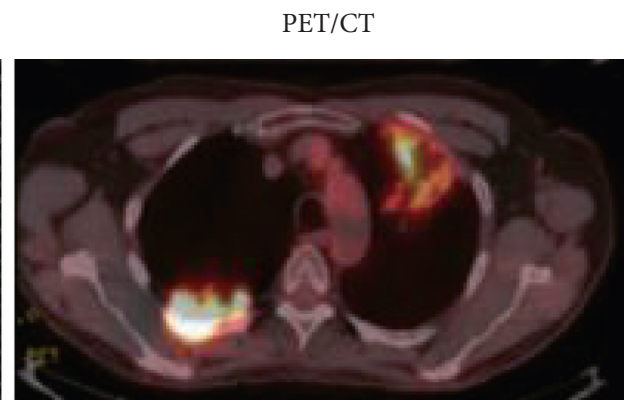

(c)

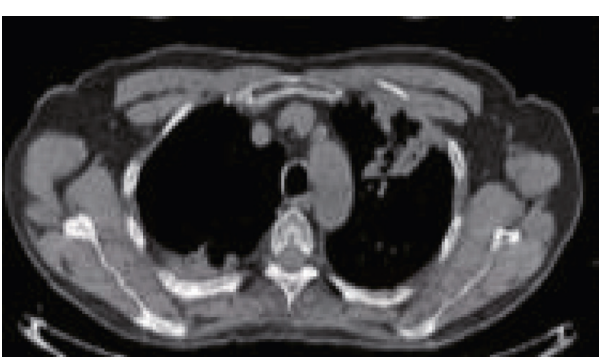

Figure 3: Radiotherapy plans corresponding to different target areas: (a) PET/CT and (b) CT.

TABLE 2: Comparison of normal tissue dose distribution parameters of different radiotherapy plans.

\begin{tabular}{lccrr}
\hline Dose distribution parameters & CT & PET/CT & $t$ value & $P$ value \\
\hline Double lungs V20 (\%) & $26.45 \pm 4.72$ & $23.43 \pm 5.11$ & 6.668 & $<0.01$ \\
Double lungs V30 (\%) & $19.46 \pm 3.07$ & $17.76 \pm 3.41$ & 2.299 & $<0.05$ \\
Spinal cord maximum dose (cGy) & $3654 \pm 468$ & $3447 \pm 420$ & 1.258 & $>0.05$ \\
Cardiac mean dose (cGy) & $1682 \pm 937$ & $1619 \pm 903$ & 0.725 & $>0.05$ \\
Esophageal average dose (cGy) & $3057 \pm 548$ & $2839 \pm 661$ & 0.916 & $>0.05$ \\
\hline
\end{tabular}

avoid geometric loss, it needs to be carried out along the outer edge of the fusion lesion, so that the target area can be delineated using PET or CT images alone. Also, time may affect the dose distribution. The author's research shows that when PET-CT fusion images are used to delineate the target area, the dose difference between the lung dose (V20, average lung dose), heart, and spinal cord is statistically significant. The increase in the above normal tissue dose is mainly because the volume of the target area outlined by different images is different; PTV compared with PET + CT group is the largest in the three groups of plans, so the normal tissues, especially the lungs, are also the highest in this group of plans. According to the grouping analysis of the lesions in different positions (upper and lower lungs), there was no statistically significant difference in the V20 of the upper lung lesions in PET, CT, and PET + CT groups, while the difference in the three groups of lower lung lesions was obvious. The main purpose of PET image in the outline of the target area is to better find the boundary between active tumor cells and nontumor tissue and to supplement and modify the target area outlined by CT anatomical images. Because the two images are essentially different, in order to minimize the impact of inconsistent metabolic information and anatomical information in the lesion, the study mainly selected lesions with clear borders, no atelectasis, no inflammation, and obvious FDG uptake. 
The difference of GTV-PET is not statistically significant, so we can focus on the analysis of the influence of respiratory motion on the fusion accuracy of the two. Because the current radiotherapy planning system is based on CT, although the use of PET images for delineation can reduce the amount of normal tissue [4], there is also a risk of geometric loss, because there is still no pathological verification study on PET and CT delineation of the target area. Reporting requires further in-depth research.

Although PET/CT has shown important value in the design of tumor radiotherapy plans, due to the long acquisition time of PET/CT, severely affected by breathing and heartbeat, and low image resolution, the application of PET/ $\mathrm{CT}$ in radiotherapy is limited. The low image contrast makes it difficult to distinguish the tumor target area from the surrounding tissue. Even for experienced clinicians, manually segmenting PET/CT images is difficult and timeconsuming. Therefore, there is an urgent clinical need for automatic segmentation of PET/CT image target areas. Wu [5] compared three methods for delineating lung cancer target area: $40 \%$ threshold method, SUV $=2.5$ delineation method, and formula method, but these methods are not universal. PCNN can produce a recognition effect similar to human vision and is suitable for low-resolution image segmentation. Therefore, this paper uses an image segmentation method based on the PCNN model that is independently written to automatically segment PET/CT images. The average GTV-PET auto result was $(142.54 \pm 120.73) \mathrm{cm} 3$, and the average GTV-PET manually delineated target area was $(146.20 \pm 101.32) \mathrm{cm} 3$. The difference between the two is not statistically significant $(P>0.05)$, indicating that this method is reliable and accurate.

The author's research results initially show that even if the machine system error is only within the range of $0.8 \mathrm{~mm}$, and the specific fixation device is used to limit the movement of the subject during the examination, the subject performs PET-CT examination while calmly breathing. However, there is still a certain deviation between the fusion of PET images and CT images of lung lesions, and the difference in fusion accuracy between upper lung lesions and lower lung lesions is not obvious and needs further research to confirm. Therefore, more attention should be paid to the influence of fusion accuracy when using PET-CT fusion images to formulate radiotherapy plans for specific lung lesions as follows. Due to the influence of breathing motion, the PET-CT same-machine image has a certain deviation in the display of the NSCLC target area. In order to improve the accuracy of PET image functional target area delineation, the clinical application of PET-CT needs to introduce four-dimensional gating technology when formulating a radiotherapy plan or use a CT scan under a specific breathing state, and use the PET image and CT image fusion of the corresponding respiratory movement phase to delineate the target area, more realistically reflect the condition of the lesion in the human body, further improve the accuracy of radiotherapy, and improve the dose laying the foundation. Zbib et al. [6] studied the effect of breathing dynamics on PET-CT chest fusion images. Patients underwent CT scans during calm breathing, maximum end- expiratory breath holding, maximum end-expiratory breath holding, and normal end-expiratory breath holding. It is $-11.6,-44.4,-9.5$, and $0.4 \mathrm{~mm}$, indicating that the normal end-tidal breath-holding CT scan for the same-machine image fusion system or the different-machine system caused the smallest breathing dynamics. Zhang et al. [7] reported that the use of gated technology for FDG PET imaging of lung cancer can reduce the tumor volume by $28 \%$ and the standard uptake value by $56.5 \%$, while with the use of respiratory gated PET-CT scan PET and CT images of the lesion COG, the distance can be reduced to below $5 \mathrm{~mm}$ [8], which is more conducive for accurately delineating the target area and protecting normal tissues to the greatest extent.

PET/CT imaging has important clinical value in determining the range of lung cancer target area. Tran and $\mathrm{Wu}$ [1] delineated tumor target areas based on PET/CT images and CT images of 36 lung cancer patients and found that 21 cases of radiotherapy target areas changed to varying degrees. In 12 cases, the target area decreased due to the distinction between tumor and atelectasis, and in 7 cases, the target area increased. Xu et al. [2] analyzed 92 cases of lung cancer patients who underwent PET/CT positioning scan before radiotherapy and found that 45 cases had significant changes. This study found that the target area obtained by PET/CT image segmentation program GTV-PET auto $<$ CT manually delineated the target area. The average value of GTV-PET auto is $(142.54 \pm 120.73) \mathrm{cm} 3$, and the average value of GTV-CT is $(161.43 \pm 139.30) \mathrm{cm} 3$. The difference between the two is significant and statistically significant $(P<0.05)$. The reason for shrinking the target area in 8 out of 12 patients is that $\mathrm{PET} / \mathrm{CT}$ images distinguish tumor and atelectasis tissues well. This study shows that PET/CT delineation of lung cancer target area is more accurate than simple CT image delineation, suitable for precise radiotherapy, and can reduce normal tissue damage.

PET/CT images bring about changes in the tumor target area, which will inevitably affect the radiotherapy plan and the dose of tumor and normal tissue exposure. The results of Rios Velazquez et al. [3] showed that, compared with traditional CT, PET-guided radiotherapy planning increased 15 cases of lung V20 and decreased 22 cases; 8 cases of cardiac V36 increased and 14 cases decreased. Gallivanone et al. [4] showed that the target area change caused by PET/CT significantly changed the dose distribution of normal tissues. The decrease in GTV led to a decrease in lung and esophageal dose, and the increase in GTV resulted in an increase in lung and esophagus dose. In this paper, two different IMRT plans were developed based on PET/CT automatic segmentation target and CT outlined target areas, and it was found that both lungs had different dose gradients V20 and V30 in the PET/CT automatic segmentation targetguided radiotherapy plan, which were significantly reduced. Among them, V20 decreased from $(26.45 \pm 4.72) \%$ to $(23.43 \pm 5.11) \%$, and V30 decreased from $(19.46 \pm 3.07) \%$ to $(17.76 \pm 3.41) \%$, the differences were statistically significant $(P<0.05)$. The above results indicate that the average radiation dose received by normal tissues of 12 patients has decreased, and it does not mean that the weight of normal tissues of all patients has decreased. Ten of the 12 patients in 
this article are in line with the above conclusions, and the other two are not significantly different. The results of this paper show that the automatic PET image segmentation method can affect the radiotherapy plan and normal tissue intake through more accurate tumor target positioning, reduce the incidence of lung cancer complications, and help guide the increase of radiation dose.

\section{Conclusion}

PET/CT images help to accurately define the boundaries of the radiotherapy target area and lay the foundation for precise radiotherapy. Fast and accurate segmentation of $\mathrm{PET} / \mathrm{CT}$ target area is the key to precise radiotherapy. This article discusses the role of PET/CT image segmentation technology in tumor target area determination and radiotherapy planning. Preliminary research shows that this technique can improve the local control rate of tumors and reduce the incidence of normal tissue complications. Further research on PET/CT image automatic segmentation technology suitable for clinical practice will play an important role in precise radiotherapy for lung cancer.

\section{Data Availability}

The data used to support the findings of this study are available from the corresponding author upon request.

\section{Conflicts of Interest}

The authors declare no conflicts of interest.

\section{Acknowledgments}

This work was supported by the National Natural Scientific Foundation of China (Grant no. 81903125) and the Natural Science Foundation of Guangdong Province, China (Grant no. 2018A030310513).

\section{References}

[1] D. C. Tran and Z. Wu, "A new approach of dynamic clustering based on particle swarm optimization and application in image segmentation," Computing and Informatics, vol. 36, no. 3, pp. 637-663, 2017.

[2] Y. Xu, S. Wu, M. Wang, and Y. Zou, "Design and implementation of distributed RSA algorithm based on Hadoop," Journal of Ambient Intelligence and Humanized Computing, vol. 11, pp. 1047-1053, 2020.

[3] E. Rios Velazquez, R. Meier, W. D. Dunn Jr et al., "Fully automatic gbm segmentation in the tcga-gbm dataset: prognosis and correlation with vasari features," Entific Reports, vol. 5, no. 4, Article ID 16822, 2015.

[4] F. Gallivanone, M. Interlenghi, C. Canervari, and I. Castiglioni, "A fully automatic, threshold-based segmentation method for the estimation of the metabolic tumor volume from pet images: validation on $3 \mathrm{~d}$ printed anthropomorphic oncological lesions," Journal of Instrumentation, vol. 11, no. 1, Article ID C01022, 2016.

[5] S. Wu, "A traffic motion object extraction algorithm," International Journal of Bifurcation and Chaos, vol. 25, no. 14, Article ID 1540039, 2015.
[6] H. Zbib, S. Mouysset, S. Stute et al., "Unsupervised spectral clustering for segmentation of dynamic pet images," IEEE Transactions on Nuclear Science, vol. 62, no. 3, pp. 840-850, 2015.

[7] G. Zhang, D. Han, C. Ma et al., "Gradient-based delineation of the primary gtv on flt pet in squamous cell cancer of the thoracic esophagus and impact on radiotherapy planning," Radiation Oncology, vol. 10, no. 1, pp. 11-17, 2015.

[8] S. Wu, M. Wang, and Y. Zou, "Research on internet information mining based on agent algorithm," Future Generation Computer Systems, vol. 86, pp. 598-602, 2018. 